$181-183^{\circ}$ auf. Ausbeute 6 g (49,8\% d. Th.). Der Metall-Wasserstoff-Austausch errechnet sich aus 1,16 g $\mathrm{C}_{2} \mathrm{H}_{4} \mathrm{NLi}$ und $4,81 \mathrm{~g}\left(\mathrm{C}_{6} \mathrm{H}_{11}\right)_{2} \mathrm{PH}$ zu 48,3 Prozent. III gleicht in seinen Löslichkeitseigenschaften I. $\mathrm{H}_{2} \mathrm{~N}-\mathrm{CH}_{2}-\mathrm{CH}_{2}-\mathrm{P}\left(\mathrm{C}_{6} \mathrm{H}_{11}\right)_{2} \quad(241,1)$ Ber. P 12,85, Gef. P 12,75.

[2-Methylammonium jodid - äthyl]-diphenylphosphin (IV): $4,6 \mathrm{~g}$ I werden in $20 \mathrm{~cm}^{3}$ Äther gelöst und mit $5,7 \mathrm{~g} \mathrm{CH}_{3} \mathrm{~J}$ in $20 \mathrm{~cm}^{3}$ Äther versetzt. Das Reaktionsgemisch wird kurz unter Rückfluß gekocht und 24 Stdn. stehen gelassen, wobei IV allmählich auskristallisiert. IV wird über eine G3-Fritte abfiltriert, mit Äther gewaschen und aus Äther/Äthanol umkristallisiert. Ausbeute $6,4 \mathrm{~g}(86,3 \% \mathrm{~d}$. Th. $)$; Zers.-P. $146-148^{\circ}$. IV ist luftbeständig, aber hygroskopisch und löst sich gut in Äthanol und Wasser.
$\left[\left(\mathrm{C}_{6} \mathrm{H}_{5}\right)_{2} \mathrm{P}-\mathrm{CH}_{2}-\mathrm{CH}_{2}-\mathrm{N}^{\oplus} \mathrm{H}_{2} \mathrm{CH}_{3}\right] \mathrm{J}^{\ominus}(371,1)$ Ber. P 8,35 J 34,21 , Gef. P 8,59 J 34,28.

[2-Äthylammoniumjodid-äthyl]-diäthylphosphin $(V)$ : Analog IV erhält man aus 2,5 g II und 5,62 $\mathrm{g} \mathrm{C}_{2} \mathrm{H}_{5} \mathrm{~J}$ in $20 \mathrm{~cm}^{3}$ Äther nach üblicher Aufarbeitung $4,8 \mathrm{~g} \mathrm{~V}$ $(88,7 \%$ d. Th. $)$; Zers.-P. $80^{\circ}$. V besitzt die gleichen Eigenschaften wie IV.

$\left[\left(\mathrm{C}_{2} \mathrm{H}_{5}\right)_{2} \mathrm{P}-\mathrm{CH}_{2}-\mathrm{CH}_{2}-\mathrm{N}^{\oplus} \mathrm{H}_{2} \mathrm{C}_{2} \mathrm{H}_{5}\right] \mathrm{J}^{\ominus}(288,9)$

Ber. P 10,72 J 43,92, Gef. P 10,87 J 44,05.

[2-Methylammoniumjodid - äthyl]- dicyclohexylphosphin $(V I)$ : Wie zuvor entstehen aus 2,4 g III und 2,8 $\mathrm{g}$ $\mathrm{CH}_{3} \mathrm{~J}$ in $60 \mathrm{~cm}^{3}$ Atther $3 \mathrm{~g}$ VI $(78,3 \%$ d. Th.) ; Zers.-P. $94-96^{\circ}$. Die Eigenschaften von VI gleichen IV.

$\left[\left(\mathrm{C}_{6} \mathrm{H}_{11}\right)_{2} \mathrm{P}-\mathrm{CH}_{2}-\mathrm{CH}_{2}-\mathrm{N}^{\oplus} \mathrm{H}_{2} \mathrm{CH}_{3}\right] \mathrm{J} \odot(383,1)$

Ber. P 8,09 J 33,13 , Gef. P 8,14 J 33,21.

\section{Die Darstellung von Heptaschwefelimid aus Chlorsulfan und Ammoniak}

F. FehÉr, R. KREUtz und Fr.-R. Minz ${ }^{2}$

Institut für Anorganische Chemie der Universität zu Köln

(Z. Naturforschg. 20 b, 918-919 [1965]; eingegangen am 7. Juli 1965)

Die bisher in der Literatur beschriebenen Methoden zur präparativen Darstellung von $\mathrm{S}_{7} \mathrm{NH}$ gehen alle von $\mathrm{S}_{2} \mathrm{Cl}_{2}$ aus, das in verschiedenen Lösungsmitteln bei verschiedenen Temperaturen mit $\mathrm{NH}_{3}$ umgesetzt wird ${ }^{3-9}$. Dabei tritt offenbar unter dem Einfluß des Ammoniaks eine Kettenverlängerung ein, die nach Erreichen von acht Gliedern zu einem Ringschluß führt ${ }^{10}$. Bei dieser Reaktion entsteht immer auch Tetraschwefeltetranitrid, das offenbar aus dem $\mathrm{SCl}_{2}$ gebildet wird, welches bei der Kettenverlängerung auftritt. Es lag nahe, diese in etwas unübersichtlicher Reaktion verlaufende Verlängerung durch Anwendung höherer Chlorsulfane, die von vorneherein eine längere Schwefelkette enthalten, zu umgehen, und so eine Verbesserung der Ausbeute an $\mathrm{S}_{7} \mathrm{NH}$, bezogen auf den eingesetzten Schwefel, zu erzielen.

Unter Berücksichtigung und entsprechender Modifizierung der in der Literatur gegebenen Arbeitsvor-

1 77. Mitt.: H. D. Lutz, Z. anorg. allg. Chem., im Druck.

2 Teil der Dissertation, Köln 1965.

3 M. H. M. Arnold. J. A. C. Hugill u. J. M. Huston, J. chem. Soc. [London] 1936, 1645.

4 M. H. M. Annold, U. S. Pat. 2.372.046 [1945].

5 M. Goehring, H. Herb u. W. Косн, Z. anorg. allg. Chem. 264, 137 [1951].

6 A. Meuwsen u. F. Schlossnagel, Z. anorg. allg. Chem. 271, 226 [1953].

7 V. Brustier u. Gracia-Fernandez, Bull. Soc. chim. France (5) $1955,1299$.

8 M. Becke-Goehring, H. Jenne u. E. Fluck, Chem. Ber. 91, 1947 [1958].

9 S. Milagros Villena-Blanco, UCRL - 11081 M. S. Thesis.

10 Ein von M. Becke-Goehring vorgeschlagener Reaktionsablauf (Chem. Ber., loc. cit.) sieht die Bildung eines Adduk- schriften haben wir die höheren Chlorsulfate $\mathrm{S}_{\mathrm{x}} \mathrm{Cl}_{2}$ $(x=3 ; 4 ; 5 \text { und } 7)^{11}$ mit $\mathrm{NH}_{3}$ umgesetzt und ebenfalls Heptaschwefelimid erhalten. Tab. 1 zeigt die durchschnittlichen Ausbeuten bei den verschiedenen, in zahlreichen Versuchen eingesetzten Chlorsulfanen, ein-

\begin{tabular}{|c|c|c|}
\hline Chlorsulfan & $\begin{array}{c}\text { Ausbeute } \\
\text { bez. a. R.-Gl. } \\
{[\%]}\end{array}$ & $\begin{array}{c}\text { bez. a. S } \\
{[\%]}\end{array}$ \\
\hline $\mathrm{S}_{2} \mathrm{Cl}_{2}$ & 37 & 21,5 \\
$\mathrm{~S}_{3} \mathrm{Cl}_{2}$ & 40 & 30 \\
$\mathrm{~S}_{2} \mathrm{Cl}_{2}$ & 20 & 17 \\
$\mathrm{~S}_{5} \mathrm{Cl}_{2}$ & 12 & 11 \\
$\mathrm{~S}_{7} \mathrm{Cl}_{2}$ & 30 & 30 \\
\hline
\end{tabular}

Tab. 1. Ausbeute an $\mathrm{S}_{7} \mathrm{NH}$.

mal bezogen auf die Reaktionsgleichungen (1) - (5), zum anderen bezogen auf den eingesetzten Schwefel.

$$
\begin{array}{rr}
6 \mathrm{~S}_{2} \mathrm{Cl}_{2}+\mathrm{NH}_{3} \rightarrow \mathrm{S}_{7} \mathrm{NH}+5 \mathrm{SCl}_{2}+2 \mathrm{HCl} \\
3 \mathrm{~S}_{3} \mathrm{Cl}_{2}+\mathrm{NH}_{3} \rightarrow \mathrm{S}_{7} \mathrm{NH}+2 \mathrm{SCl}_{2}+2 \mathrm{HCl} \\
2 \mathrm{~S}_{4} \mathrm{Cl}_{2}+\mathrm{NH}_{3} \rightarrow \mathrm{S}_{7} \mathrm{NH}+\mathrm{SCl}_{2}+2 \mathrm{HCl} \\
6 \mathrm{~S}_{5} \mathrm{Cl}_{2}+4 \mathrm{NH}_{3} \rightarrow 4 \mathrm{~S}_{7} \mathrm{NH}+2 \mathrm{SCl}_{2}+8 \mathrm{HCl} \\
\mathrm{S}_{7} \mathrm{Cl}_{2}+\mathrm{NH}_{3} \rightarrow \mathrm{S}_{7} \mathrm{NH}+2 \mathrm{HCl}
\end{array}
$$

tes von $\mathrm{NH}_{3}$ mit $\mathrm{S}_{2} \mathrm{Sl}_{2}$ vor:

$\mathrm{S}_{2} \mathrm{Cl}_{2}+\mathrm{NH}_{3} \rightarrow\left(\mathrm{Cl}-\mathrm{S}_{2}-\mathrm{NH}_{3}\right) \mathrm{Cl}^{\ominus}$, das dann unter $\mathrm{SCl}_{2}$. Austritt mit überschüssigem Dichlordisulfan weiterreagiert :

$\left(\mathrm{Cl}-\mathrm{S}_{2}-\mathrm{NH}_{3}\right) \mathrm{Cl}{ }^{\ominus}+\mathrm{S}_{2} \mathrm{Cl}_{2} \rightarrow\left(\mathrm{Cl}-\mathrm{S}_{3}-\mathrm{NH}_{3}\right) \mathrm{Cl}^{\ominus}+\mathrm{SCl}_{2}$;

$\left(\mathrm{Cl}-\mathrm{S}_{3}-\mathrm{NH}_{3}\right) \mathrm{Cl}^{\ominus}+\mathrm{S}_{2} \mathrm{Cl}_{2} \rightarrow\left(\mathrm{Cl}_{-}-\mathrm{S}_{4}-\mathrm{NH}_{3}\right) \mathrm{Cl}{ }^{\ominus}+\mathrm{SCl}_{2} ;$ usw. bis schließlich $\left(\mathrm{Cl}^{-} \mathrm{S}_{7}-\mathrm{NH}_{3}\right) \mathrm{Cl}^{\ominus}$ gebildet wird, aus dem unter Ringschluß $\mathrm{S}_{7} \mathrm{NH}$ entsteht. Milagros (loc. cit.) stellt auf Grund seiner Versuchsreihe zur Darstellung von Heptaschwefelimid aus $\mathrm{S}_{2} \mathrm{Cl}_{2}$ diesen Reaktionsmechanismus in Frage.

11 F. FehÉr, K. Naused u. He. Weber, Z. anorg. allg. Chem. 290, 303 [1957]; F. FEHÉR, u. S. Ristić, Z. anorg. allg. Chem. 293, 307 [1958]; F. FeHÉR, H. Goebell u. FR.-R. Minz, Z. anorg. allg. Chem., im Druck. 
Die Tatsache, daß die Ausbeute an $\mathrm{S}_{7} \mathrm{NH}$ nicht über ca. $40 \%$ ansteigt, erklärt sich daraus, daß $\mathrm{S}_{7} \mathrm{NH}$ unter der Einwirkung von $\mathrm{NH}_{3}$ einen alkalischen Abbau erleidet ${ }^{12}$. Läßt man z. B. das ausreagierte Umsetzungsgut bei einem $p_{\mathrm{H}}$ von $>8$ länger als sechs Stdn. ohne Aufarbeitung stehen, so erhält man überhaupt kein $\mathrm{S}_{7} \mathrm{NH}$. Die Ergebnisse der Untersuchungen über die

12 S. Mrlagros (loc. cit.) sieht den Grund für die geringe Ausbeute in der Reaktion von $\mathrm{S}_{2} \mathrm{Cl}_{2}$ mit $\mathrm{S}_{7} \mathrm{NH}$ zu $\mathrm{S}_{2}\left(\mathrm{~S}_{7} \mathrm{~N}\right)_{2}$,
Kinetik dieses baseninduzierten Abbaus von $\mathrm{S}_{7} \mathrm{NH}$ werden wir an anderer Stelle veröffentlichen.

Es sei die interessante zusätzliche Beobachtung vermerkt, daß die Ausbeute an $\mathrm{S}_{4} \mathrm{~N}_{4}$ mit wachsender Kettenlänge des Chlorsulfans deutlich abnimmt und bei Verwendung von $\mathrm{S}_{7} \mathrm{Cl}_{2}$ fast völlig fehlt.

Die relativ geringe Ausbeute an $\mathrm{S}_{7} \mathrm{NH}$ bei Verwendung von $\mathrm{S}_{5} \mathrm{Cl}_{2}$ erklärt sich daraus, daß eine Kettenverlängerung aus 2 Molekülen Dichlorpentasulfan bereits eine Kette liefert, die mehr als 7 S-Atome enthält. $\mathrm{Da} ß$ überhaupt Heptaschwefelimid entsteht, ist auf eine Disproportionierung des Chlorsulfans zurückzuführen.

\section{Rhythmische katalytische Mutationen der Ionen $\mathrm{Cu}^{2 \oplus}$ und $\left[\mathrm{Fe}(\mathrm{CN})_{6}\right]^{4 \odot}$ auf Eisen(III)-hydroxid-Mischträgern verschiedener Zusammensetzung}

Alfons Krause, F. Domka und B. Marciniec

Institut für anorganische Chemie der Universität Poznań (Polen)

(Z. Naturforschg. 20 b, 919-920 [1965]; eingegangen am 21. Mai 1965)

Beim Auftragen von zwei verschiedenen Ionen auf einem Träger lassen sich, wenn es sich um die katalytische Aktivität solcher Träger/Ionen-Katalysatoren handelt, drei Fälle unterscheiden ${ }^{1}$. Entweder können sich $\mathrm{Ion}_{1}$ und $\mathrm{Ion}_{2}$ additiv bzw. superadditiv verstärken oder auch gegenseitig in ihrer Wirkung abschwächen (katalytischer Ionenantagonismus). Der dritte Fall betrifft die zeitliche Reihenfolge, in welcher $\mathrm{Ion}_{1}$ und $\mathrm{Ion}_{2}$ mit dem Träger in Berührung kommen, wobei gegebenenfalls verschieden aktive Mehrstoffkatalysatoren in Gestalt von Träger + Ion $_{1}+$ Ion $_{2}$ oder Träger + $\mathrm{Ion}_{2}+\mathrm{Ion}_{1}$ entstehen können. Diese katalytische $\mathrm{Mu}$ tation ist, obwohl nur auf bestimmte Fälle von Trägern und Ionen beschränkt, in den letzten Jahren mehrfach beschrieben worden ${ }^{2}$. Hier wird über ein neues Beispiel berichtet, das die Ionen $\mathrm{Cu}^{2 \oplus}$ und $\left[\mathrm{Fe}(\mathrm{CN})_{6}\right]^{4 \ominus}$ betrifft, falls diese auf einem Eisen(III)-hydroxidMischträger untergebracht werden, der aus $\gamma$ - $\mathrm{FeOOH}$ und dem röntgenamorphen Eisen(III)-hydroxid besteht. Als Substrat diente eine $\mathrm{HCOOH} / \mathrm{H}_{2} \mathrm{O}_{2}$-Mischlösung. Wie aus Tab. 1 ersichtlich, wird die Ameisensäure in Gegenwart der vorliegenden Träger/Ionen-Katalysatoren mit einer verschiedenen Geschwindigkeit zu $\mathrm{CO}_{2}$ oxydiert. Man bemerkt, daß der Katalysator: Träger + $\left[\mathrm{Fe}(\mathrm{CN})_{6}\right]^{4 \oplus}+\mathrm{Cu}^{2 \ominus}$ weit besser ist als der in der Reihenfolge: Träger $+\mathrm{Cu}^{2}{ }^{\oplus}+\left[\mathrm{Fe}(\mathrm{CN})_{6}\right]^{4 \ominus}$. Dieses Ergebnis ist allerdings von der Zusammensetzung des Trägers abhängig. Wenn diese dem Mol-Verhältnis $\gamma$-FeOOH: röntgenamorphem Eisen(III)-hydroxid (auf die Oxide gerechnet) $=1: 0,75$ entspricht, so tritt die erwähnte katalytische Mutation sehr deutlich hervor. Falls das Mol-Verh. der Trägerhydroxide sich auf $1: 0,25$ beläuft, so machen sich die Mutationserscheinungen merkwürdigerweise im umgekehrten Sinne geltend, indem nunmehr der nach dem Schema: Träger $+\mathrm{Cu}^{2 \oplus}+\left[\mathrm{Fe}(\mathrm{CN})_{6}\right]^{4 \ominus}$ erhaltene Katalysator der bevorzugte ist. Dazwischen gibt es auch, wenn nämlich das bewußte Mol-Verh. $1: 1$ oder $1: 0,5$ beträgt, praktisch mutationslose Zustände (Tab. 1). Man hat also, um kurz zu wiederholen, bei einem Mol-Verh. der Trägerhydroxide 1:1 praktisch keine Mutation, bei 1 : 0,75 Mutation, bei $1: 0,5$ keine Mutation und bei $1: 0,25$ wieder Mutation. Solche rhythmischen Er-

\begin{tabular}{|c|c|c|c|c|c|c|c|c|c|c|}
\hline \multirow{2}{*}{$\begin{array}{c}\text { Zeit } \\
\text { [min] }\end{array}$} & \multicolumn{2}{|c|}{$\operatorname{Tr} .1 \gamma: 1 \mathrm{am}$} & \multicolumn{2}{|c|}{$\operatorname{Tr} .1 \gamma: 0,75$ am. } & \multicolumn{2}{|c|}{ Tr. $1 \gamma: 0,50 \mathrm{am}}$. & \multicolumn{2}{|c|}{ Tr. $1 \gamma: 0,25$ am. } & \multirow{2}{*}{$\begin{array}{l}\mathrm{a}+\mathrm{b} \\
\text { allein } \\
\text { (oder } \\
\text { umge- } \\
\text { kehrt) }\end{array}$} & \multirow{2}{*}{$\begin{array}{l}\text { Blind- - } \\
\text { probe }\end{array}$} \\
\hline & $\stackrel{+}{a+b}$ & $\stackrel{+}{b+a}$ & $\begin{array}{l}+ \\
a+b\end{array}$ & $\stackrel{+}{+}+a$ & $\begin{array}{c}+ \\
a+b\end{array}$ & $\stackrel{+}{b+a}$ & $\stackrel{+}{a+b}$ & $\stackrel{+}{b+a}$ & & \\
\hline 0 & 25,0 & 25,0 & 25,0 & 25,0 & 25,0 & 25,0 & 25,0 & 25,0 & 25,0 & 25,0 \\
\hline 120 & 12,4 & 13,6 & 14,7 & 8,8 & 10,1 & 11,3 & 12,0 & 15,3 & 24,2 & 24,7 \\
\hline 180 & 5,2 & 6,5 & 8,5 & 1,8 & 3,5 & 3,6 & 4,1 & 8,7 & 24,0 & 24,5 \\
\hline
\end{tabular}

Tab. 1. Katalytische Mutation der Ionen $\mathrm{Cu}^{2} \oplus$ (a) und $\left[\mathrm{Fe}(\mathrm{CN})_{6}\right]^{4} \ominus$ (b) auf Eisen (III) -hydroxid-Trägern (Tr.) im System $\mathrm{HCOOH} / \mathrm{H}_{2} \mathrm{O}_{2}$ bei $37^{\circ}$. Die Bezeichnung $\gamma$ : am. bezieht sich auf das Mol.-Verh. $\gamma$-FeOOH: röntgenamorphem Eisen (III)-hydroxid. - Die Zahlenwerte geben den Verbrauch an $0,02-n \cdot \mathrm{NaOH}$ (in ml) für je $10 \mathrm{ml}$ Reaktionslösung an.

1 A. Krause, Actes IIième Congr. internat. Catalyse, Paris 1960.
2 A. Krause u. Mitarb., C. R. mens. Acad. polon. Sci., Cl. math. natur. No. 5-10, 57 [1951]; Roczniki chem. (Ann. Soc. chim. Polonorum) 29, 3 [1955]; Experientia [Basel] 20, 420 [1964]; Naturwissenschaften 51, 406 [1964]. 